\title{
Quasilinear equations with natural growth
}

\section{David Arcoya and Pedro J. Martínez-Aparicio}

\begin{abstract}
We study the existence of positive solution $w \in H_{0}^{1}(\Omega)$ of the quasilinear equation $-\Delta w+g(w)|\nabla w|^{2}=a(x), x \in \Omega$, where $\Omega$ is a bounded domain in $\mathbb{R}^{N}, 0 \leq a \in L^{\infty}(\Omega)$ and $g$ is a nonnegative continuous function on $(0,+\infty)$ which may have a singularity at zero.
\end{abstract}

\section{Introduction}

We study the existence of solutions for the quasilinear b.v.p.

$$
\begin{gathered}
-\Delta w+g(w)|\nabla w|^{2}=a(x), \quad x \in \Omega \\
w \in H_{0}^{1}(\Omega)
\end{gathered}
$$

where $\Omega$ is a bounded open set in $\mathbb{R}^{N},(N \geq 3), 0 \leq a \in L^{\infty}(\Omega)$ and $g$ is a continuous function in $(0,+\infty)$. Following the terminology of [15], we say that the quadratic growth in $\nabla w$ of the nonlinear differential operator in $(P)$ is "natural". Two reasons can be given to use this term. Firstly, the invariance of this growth under the simple change of variables $v=F(w)$, where $F$ is a smooth function ${ }^{1}$. On the other hand, recall the nonexistence result given in [22] for the case in which the growth in $\nabla w$ is faster than quadratic.

The existence of solutions of this problem for every function $a(x)$ in a given Lebesgue space has been systematically studied by Boccardo, Murat and Puel, see $[5,6]$ and references therein and by Bensoussan, Boccardo and

2000 Mathematics Subject Classification: 35J60, 35J65, 35B45.

Keywords: Quasilinear elliptic equations, critical growth, singular nonlinearity.

${ }^{1}$ On the contrary, the class of semilinear equations is unnatural in the sense that it is not invariant if one makes such a change of variable $v=F(w)$, because the resulting equation in $v$ involves $|\nabla v|^{2}$ (except in the trivial case where $F$ is linear). 
Murat [4]. They consider a continuous $g$ in $\mathbb{R}$ which does not satisfy any growth restriction with respect to $w$. In [4] they impose the sign condition $g(s) s \geq 0$, for every $s \in \mathbb{R}$. On the other hand, in $[5,6]$ the authors consider the Dirichlet boundary value problem for an equation with a more general quadratic term $f(x, w, \nabla w)$ (instead of the term $\left.g(w)|\nabla w|^{2}\right)$. In that case, they require (see (2.10) in [5] and (3.10) in [6]) a more general "one-side condition" than the sign condition. However, we point out that in the case $f(x, w, \nabla w)=g(w)|\nabla w|^{2}$ this one-side hypothesis is equivalent to the sign condition.

In [21] (see also [8]), the sign condition is not assumed and the existence of solution of $(P)$ is proved provided that the asymptotic condition

$$
\lim _{|s| \rightarrow \infty} \frac{e^{\left|\int_{0}^{s}\right| g(r)|d r|}}{1+\left|\int_{0}^{s} e^{\left|\int_{0}^{t}\right| g(r)|d r|} d t\right|}=0
$$

holds.

On the other hand, in $[12,14]$ the existence of solution of $(P)$ is proved without assuming neither the sign condition nor the asymptotic condition at infinity, but requiring that the data $a(x)$ is small enough.

Regularity of the solutions are studied in [9]. Some extensions to systems of elliptic partial differential equations are discussed in $[2,3,18]$, while the case of unbounded domains $\Omega$ is treated in [10]. More recently, the case $g \leq 0$ and decreasing is studied in [1].

The scope of this work is to study the existence of positive solution of $(P)$ in the case that $g$ may have a singularity at zero. Our simplest model is the equation

$$
-\Delta w+\frac{1}{w}|\nabla w|^{2}=a(x), \quad x \in \Omega .
$$

This kind of singular equation appears for instance if, for $p>1$, we make the change of variables $w=\int_{u}^{+\infty} d s / s^{p /(p-1)}$ in the simple semilinear equation

$$
\Delta u=a(x) u^{p /(p-1)}, \quad x \in \Omega .
$$

Recalling the invariance of the quadratic growth in $\nabla w$, we see hence the interest of studying this singular equation. We point out that, up to the authors' knowledge, existence results for singular $g$ are unknown in the literature of elliptic equations with quadratic growth in $\nabla w$. Our main result is the following. 
Theorem 1.1. Let $a \in L^{\infty}(\Omega)$ be a function satisfying

$$
\inf \{a(x) / x \in \omega\}>0, \quad \forall \omega \subset \subset \Omega .
$$

If $g:(0,+\infty) \longrightarrow[0,+\infty)$ is a continuous function such that

$$
\limsup _{s \rightarrow 0} s g(s)<+\infty
$$

then problem $(P)$ has at least one positive solution $w \in H_{0}^{1}(\Omega) \cap C^{\alpha}(\Omega)$, $(0<\alpha<1)$.

The idea to prove it consists in approximating $(P)$ by a sequence of problems $\left(P_{n}\right)$ which fall into the framework in [6] and to prove that their solutions $w_{n}$ converge to a positive solution of $(P)$. Here, due to singularity of $g, g\left(w_{n}(x)\right)$ blows up as $w_{n}(x)$ is converging to zero. This is the reason why it is not possible to apply the ideas of $[4,5,6]$ to show the strong convergence of $\nabla w_{n}$ in $L^{2}(\Omega)$ (and thus the strong convergence of the approximated solutions $w_{n}$ in $H_{0}^{1}(\Omega)$ to a solution of $(P)$ ). To overcome this difficulty, we show that $w_{n}$ are uniformly away from zero in every compact set in $\Omega$. This allows us to prove that the sequence of approximated solutions converges locally to a solution of $(P)$. The argument for the uniform estimate of $w_{n}$ is based on an a priori estimate for the subsolutions of the semilinear equation $\Delta u=a(x) u^{p}$ with $p>1$. This estimate will be proved in Proposition 2.3 by applying the result in [16] (see also [20]) about the existence of boundary blow-up solutions of the latter equation, i.e. for the semilinear problem

$$
\begin{gathered}
\Delta u=a(x) u^{p}, \text { in } \Omega \\
\lim _{\operatorname{dist}(x, \partial \Omega) \rightarrow 0} u(x)=+\infty,
\end{gathered}
$$

where $a \in L^{\infty}(\Omega)$.

As a by-product we can use the above ideas to improve the previous results in $[6,7,21]$ by showing the existence of positive solution for the problem $(P)$ under the only assumption of continuity of $g$ on $[0,+\infty)$. In particular, we remark explicitly that it does not matter the value of $g(0) \in[0,+\infty)$ (observe that the sign condition in $[4,5,6]$ implies necessarily that $g(0)=0$ ) or the behavior of $g$ at infinity. Specifically, we prove

Theorem 1.2. Consider $0 \not \equiv a \in L^{q}(\Omega)$, for some $q>\frac{N}{2}$ with $a \geq 0$ and let $g:[0,+\infty) \longrightarrow[0,+\infty)$ be a continuous function. Then problem $(P)$ has at least one positive solution $w \in H_{0}^{1}(\Omega) \cap L^{\infty}(\Omega)$. 
The rest of the paper is organized as follows: Section 2 is devoted to give some a priori estimates for the approximated problem. In Section 3, we give a detailed proof of Theorem 1.1. The proof of Theorem 1.2 is similar and we just give its outline.

Acknowledgement. The first author wants to thank very warmly Lucio Boccardo for fruitful discussions.

\section{The approximated problem}

Let us denote by $\mathcal{S}$ the Sobolev constant, i.e.

$$
\mathcal{S}=\inf _{u \in H_{0}^{1}(\Omega)-\{0\}} \frac{\|u\|^{2}}{\|u\|_{2^{*}}^{2}},
$$

with $2^{*}=2 N /(N-2),(N \geq 3)$. Also, we denote

$$
C=\mathcal{S}^{-2^{*}}\|a\|_{\infty}^{2^{*}}|\Omega|^{\frac{4}{N-2}} 2^{\frac{N}{2} \frac{N+2}{N-2}} .
$$

By the continuity of $g$ in $(0,+\infty)$ and $(1.2)$, there exists $\lambda>1$ such that

$$
g(s) \leq \frac{\lambda}{s}, \quad \forall s \in(0, C]
$$

Observe that we can rewrite the problem as

$$
\begin{gathered}
-\Delta w+\lambda h(w)|\nabla w|^{2}=a(x) \text { in } \Omega \\
w \in H_{0}^{1}(\Omega)
\end{gathered}
$$

where $h:(0,+\infty) \longrightarrow[0,+\infty)$ is defined by $h(s) \equiv g(s) / \lambda$, for every $s>0$. Thus it satisfies

$$
h(s) \leq \frac{1}{s}, \quad \forall s \in(0, C]
$$

We approximate the function $h$ by functions $h_{n}: \mathbb{R} \longrightarrow \mathbb{R}, n \in \mathbb{N}$, which are defined by

$$
h_{n}(s):= \begin{cases}h(s), & s \geq \frac{1}{n}, \\ n^{2} s^{2} h(s), & 0<s \leq \frac{1}{n}, \\ 0, & s=0, \\ -h_{n}(-s), & s<0 .\end{cases}
$$


Observe that $h_{n}$ is continuous by (1.2) and verifies

$$
\begin{array}{ll} 
& h_{n}(s) \stackrel{n \rightarrow+\infty}{\longrightarrow} h(s), \forall s>0, \\
& h_{n}(s) \leq h(s), \forall s>0, \\
\text { and } \quad & h_{n}(s) s \geq 0, \forall s \in \mathbb{R} .
\end{array}
$$

Then, by applying [6, Theorem 3.2, p. 43], there exists a solution $w_{n}$ of the approximated problem

$$
\begin{gathered}
-\Delta w+\lambda h_{n}(w)|\nabla w|^{2}=a(x) \text { in } \Omega \\
w \in H_{0}^{1}(\Omega) .
\end{gathered}
$$

Choosing $w_{n}^{-}$as test function and using that $h_{n}(s) s \geq 0$ and $a \geq 0$ we get

$$
\int_{\Omega}\left|\nabla w_{n}^{-}\right|^{2} \leq \int_{\Omega}\left|\nabla w_{n}^{-}\right|^{2}+\lambda \int_{\Omega} w_{n}^{-} h_{n}\left(w_{n}\right)\left|\nabla w_{n}^{-}\right|^{2}=\int_{\Omega} a w_{n}^{-} \leq 0
$$

i.e. $w_{n} \geq 0$. In addition $w_{n} \not \equiv 0$ because $a(x) \not \equiv 0$.

The following a priori estimate will be useful.

Lemma 2.1. Let $w_{n}$ be a solution of $\left(P_{n}\right)$.

i) If $a \in L^{q}(\Omega)$, for some $q>\frac{N}{2}$, then the sequence $\left\{w_{n}\right\}$ is bounded in $H_{0}^{1}(\Omega)$ and in $L^{\infty}(\Omega)$.

ii) If $a \in L^{\infty}(\Omega)$, then

$$
\left\|w_{n}\right\|_{\infty} \leq C, \quad \forall n \in \mathbb{N}
$$

where $C$ is given by (2.1). In addition, $w_{n} \in C^{1, \alpha}(\Omega)(0<\alpha<1)$.

Proof. i) Taking $\varphi=w_{n}$ as test function in $\left(P_{n}\right)$, and using that $h_{n}(s) s \geq 0$, $\forall s \in \mathbb{R}$ we deduce from the Hölder inequality that

$$
\int_{\Omega}\left|\nabla w_{n}\right|^{2} \leq \int_{\Omega}\left|\nabla w_{n}\right|^{2}+\lambda \int_{\Omega} h_{n}\left(w_{n}\right) w_{n}\left|\nabla w_{n}\right|^{2}=\int_{\Omega} a w_{n} \leq\|a\|_{q}\left\|w_{n}\right\|_{q^{\prime}} .
$$

Since $q>N / 2$, we have $q^{\prime}=q /(q-1)<(N / 2)^{\prime}=N /(N-2)<2^{*}$ and hence, if we divide the above inequality by $\left\|w_{n}\right\|$, we obtain from the Sobolev embedding theorem that for some $K>0$,

$$
\left\|w_{n}\right\| \leq K\|a\|_{q}, \quad \forall n \in \mathbb{N} .
$$


On the other hand, for every solution $w_{n}$ of $\left(P_{n}\right)$, the hypothesis $h_{n}(s) s \geq 0$, for every $s \in \mathbb{R}$, implies

$$
-\Delta w_{n} \leq a(x)
$$

Then using that $a \in L^{q}(\Omega)$ and $[23$, Lemme 4.1, p. 19] we derive

$$
\left\|w_{n}\right\|_{\infty} \leq \mathcal{S}^{-2^{*}}\|a\|_{q}^{2^{*}}|\Omega|^{\beta-1} 2^{2^{*} \frac{\beta}{\beta-1}} \equiv C_{q}
$$

where $\mathcal{S}$ is the Sobolev constant and $\beta=\frac{N+2}{N-2}-\frac{2^{*}}{q}$.

ii) By taking the limit as $q$ tends to $+\infty$ in the previous inequality, we yield

$$
\left\|w_{n}\right\|_{\infty} \leq \mathcal{S}^{-2^{*}}\|a\|_{\infty}^{2^{*}}|\Omega|^{\frac{4}{N-2}} 2^{\frac{N}{2} \frac{N+2}{N-2}}=C .
$$

This $L^{\infty}$-estimate of $w_{n}$ and the boundedness of $a$ allow us to apply the Theorem 1.1, p. 251 of [17] to conclude that $w_{n} \in C^{\alpha}(\Omega)$.

We devote the rest of the proof to show by using similar ideas to those in [11] that $w_{n} \in C^{1, \alpha}(\Omega)$. Applying [7, Proposition 3.8], there exists $m>2$ such that $w_{n} \in W_{\text {loc }}^{1, m}(\Omega)$. Thus, $\left|\nabla w_{n}\right|^{2} \in L_{\text {loc }}^{m / 2}(\Omega)$ which together to the $L^{\infty}$-boundedness of $w_{n}$ and $a(x)$ gives

$$
-\Delta w_{n}=-\lambda h_{n}\left(w_{n}\right)\left|\nabla w_{n}\right|^{2}+a(x) \in L_{\mathrm{loc}}^{m / 2} .
$$

Hence, the $L^{p}$-theory of Agmon, Douglis and Nirenberg [13] and the $C^{\alpha}$-smoothness of $w_{n}$ given by Lemma 2.1-ii) yield

$$
w_{n} \in W_{\mathrm{loc}}^{2, \frac{m}{2}}(\Omega) \cap C^{\alpha}(\Omega) .
$$

Using the interpolation theorem due to Miranda [19]:

$$
W_{\mathrm{loc}}^{2, s}(\Omega) \cap C^{\alpha}(\Omega) \subset W_{\mathrm{loc}}^{1, p}(\Omega), \quad p=\frac{s(2-\alpha)-\alpha}{1-\alpha}>2 s>2,
$$

we deduce that

$$
w_{n} \in W_{\mathrm{loc}}^{1, p_{1}}(\Omega),
$$

with $p_{1}=\frac{\frac{m}{2}(2-\alpha)-\alpha}{1-\alpha}>m>2$. Returning to (2.4), we get

$$
-\Delta w_{n}=-\lambda h_{n}\left(w_{n}\right)\left|\nabla w_{n}\right|^{2}+a(x) \in L_{\mathrm{loc}}^{p_{1} / 2}
$$

and again by the $L^{p}$-theory we obtain $w_{n} \in W_{\text {loc }}^{2, \frac{p_{1}}{2}}(\Omega)$.

By iterating the process, we derive that

$$
w_{n} \in W_{\mathrm{loc}}^{1, p_{j}}(\Omega),
$$

with $p_{j}=\frac{\frac{p_{j-1}}{2}(2-\alpha)-\alpha}{1-\alpha}>p_{j-1}>2, j=2,3, \ldots$ 
Taking into account that $p_{j}$ is converging to $+\infty$, we have that

$$
w_{n} \in W_{\mathrm{loc}}^{2, p}(\Omega), \quad \forall p>2,
$$

and hence, by the embedding of $W_{\text {loc }}^{2, p}(\Omega)$ into $C^{1, \alpha}(\Omega)$ if $p \geq N$, we conclude that

$$
w_{n} \in C^{1, \alpha}(\Omega) .
$$

Proposition 2.2. Let $w_{n}$ be a solution of $\left(P_{n}\right)$. If $a(x) \in L^{\infty}(\Omega)$ satisfies (1.1), then

$$
w_{n}(x)>0, \quad \forall x \in \Omega .
$$

Proof. Fix $x_{0} \in \Omega$ and choose $R>0$ such that the sphere centered at $x_{0}$ with radius $R, B=B\left(x_{0}, R\right) \subset \subset \Omega$. From Lemma 2.1, $w_{n} \in C^{1, \alpha}(\bar{B})$ and thus there exists $K_{n}>0$ such that

$$
\left|\nabla w_{n}(x)\right|^{2} \leq K_{n}, \quad \forall x \in \bar{B} .
$$

On the other hand, taking $\widetilde{K_{n}}=\sup _{s \in(0, C]} h_{n}(s) / s,(C$ given by $(2.1))$, we also have

$$
h_{n}\left(w_{n}(x)\right) \leq \widetilde{K_{n}} w_{n}(x), \quad \forall x \in \Omega .
$$

Consequently, there exists $K>>0$ (depending on $n$ ) such that

$$
\begin{gathered}
-\Delta w_{n}+K w_{n} \geq-\Delta w_{n}+\lambda h_{n}\left(w_{n}\right)\left|\nabla w_{n}\right|^{2}=a(x) \geq 0, \quad x \in B, \\
w_{n} \geq 0, \quad \partial B .
\end{gathered}
$$

By hypothesis (1.1), $a \not \equiv 0$ in $B$ and hence the strong maximum principle implies $w_{n}>0$ in $B$. Therefore $w_{n}\left(x_{0}\right)>0$.

Now, we are going to prove that the sequence of approximated solutions are uniformly away from zero in every compact set in $\Omega$. This will be a consequence of the following

Proposition 2.3. Let $\bar{f}:[0,+\infty) \longrightarrow[0,+\infty)$ be a nondecreasing continuous function such that $\bar{f}(t)>0$ for $t>0$ and

$$
\int_{a}^{+\infty} \frac{d x}{\sqrt{\int_{a}^{x} \bar{f}(z) d z}}<+\infty, \quad \forall a>0 .
$$

Assume also that the function a satisfies (1.1). Then for every open set $\Omega_{0} \subset \subset \Omega$ there exists a positive constant $C_{\Omega_{0}}$ such that for every $u \in C(\Omega) \cap$ $H^{1}(\Omega)$ satisfying

$$
\Delta u \geq a(x) \bar{f}(u), \quad x \in \Omega
$$

it holds

$$
u(x) \leq C_{\Omega_{0}}, \quad \forall x \in \Omega_{0} .
$$


Proof. Consider $\Omega_{0} \subset \subset \Omega$. Choose a open set $\omega$ in $\Omega$ such that $\Omega_{0} \subset \subset$ $\omega \subset \subset \Omega$. By (1.1),

$$
a_{0} \equiv \inf \{a(x) / x \in \omega\}>0 .
$$

Since $u \in C(\Omega)$, we also have

$$
\alpha_{0} \equiv \max _{\bar{\omega}} u<+\infty
$$

For $\alpha \geq \alpha_{0}$, taking into account that $\bar{f}$ is nondecreasing, it is proved the existence and uniqueness of solution $v_{\alpha}$ for the b.v.p.

$$
\begin{gathered}
\Delta v_{\alpha}(x)=a_{0} \bar{f}\left(v_{\alpha}(x)\right), \quad x \in \omega \\
v_{\alpha}(x)=\alpha, \quad x \in \partial \omega .
\end{gathered}
$$

Using that $\bar{f} \geq 0$ and the definition of $\alpha_{0}$, we deduce for every $\alpha \geq \alpha_{0}$ that

$$
\begin{gathered}
\Delta\left(v_{\alpha}-u\right) \leq a_{0} \bar{f}\left(v_{\alpha}(x)\right)-a(x) \bar{f}(u) \leq a_{0}\left[\bar{f}\left(v_{\alpha}(x)\right)-\bar{f}(u)\right], \quad x \in \omega \\
v_{\alpha}-u=\alpha-u \geq 0, \quad x \in \partial \omega,
\end{gathered}
$$

and, applying the maximum principle, we have that

$$
u \leq v_{\alpha} \text { in } \omega .
$$

Now, by [16] (see also [20]), $v_{\alpha}(x)$ is nondecreasing in $\alpha$ and pointwise convergent in $\omega$ to a solution $v_{\omega}$ for the b.v.p.

$$
\begin{gathered}
\Delta v_{\omega}(x)=a_{0} \bar{f}\left(v_{\omega}(x)\right), \quad x \in \omega, \\
\lim _{\operatorname{dist}(x, \partial \omega) \rightarrow 0} v_{\omega}(x)=+\infty .
\end{gathered}
$$

Therefore $u \leq v_{\omega}$ in $\omega$ and, in particular, recalling that $\Omega_{0} \subset \subset \omega$,

$$
u(x) \leq v_{\omega}(x) \leq \max _{\overline{\Omega_{0}}} v_{\omega} \equiv C_{\Omega_{0}}, \quad x \in \Omega_{0} .
$$

Remark 2.4. The hypothesis (2.5) is usually called the Keller-Osserman condition and was introduced in [16, 20] for the study of the problem (1.3).

In the sequel, we denote

$$
\bar{f}_{\lambda}(s)=s^{\lambda^{\prime}}, \quad\left(\lambda^{\prime} \equiv \frac{\lambda}{\lambda-1}>1\right) .
$$


Observe that for every $a>0$ it holds

$$
\int_{a}^{+\infty} \frac{d s}{\bar{f}_{\lambda}(s)}<\int_{0}^{+\infty} \frac{d s}{\bar{f}_{\lambda}(s)}=+\infty
$$

This allows us to define the nonincreasing function $H_{\lambda}:(0,+\infty) \longrightarrow$ $(0,+\infty)$ given by

$$
H_{\lambda}(t)=\int_{t}^{+\infty} \frac{d s}{\bar{f}_{\lambda}(s)}
$$

Lemma 2.5. Consider the functions $\bar{f}_{\lambda}$ and $H_{\lambda}$ given respectively by (2.6) and (2.7). If $0<w \in H_{0}^{1} \cap C(\Omega)$ is a supersolution of

$$
-\triangle w+\lambda \frac{1}{w}|\nabla w|^{2}=a(x), \quad x \in \Omega
$$

then $u=H_{\lambda}^{-1}(w)$ is a subsolution of

$$
\Delta u=a(x) \bar{f}_{\lambda}(u), \quad x \in \Omega .
$$

Proof. Suppose that $w \in H_{0}^{1}(\Omega) \cap C(\Omega)$ is a supersolution of

$$
-\triangle w+\lambda \frac{1}{w}|\nabla w|^{2}=a(x), \quad x \in \Omega
$$

i.e.,

$$
\int_{\Omega} \nabla w(x) \nabla \varphi(x)+\int_{\Omega} \lambda \frac{1}{w(x)} \varphi(x)|\nabla w(x)|^{2} \geq \int_{\Omega} a(x) \varphi(x),
$$

for every $\varphi \in C_{0}^{\infty}(\Omega)$, with $\varphi \geq 0$.

Observe that by the chain rule,

$$
\varphi=\bar{f}_{\lambda}(u) \psi \in H_{0}^{1}(\Omega), \quad \forall \psi \in C_{0}^{\infty}(\Omega)
$$

and thus, choosing it as test function,

$$
\int_{\Omega} \nabla w\left[\bar{f}_{\lambda}^{\prime}(u) \nabla u \psi+\bar{f}_{\lambda}(u) \nabla \psi\right]+\lambda \int_{\Omega} \frac{1}{w} \bar{f}_{\lambda}(u) \psi|\nabla w|^{2} \geq \int_{\Omega} a \bar{f}_{\lambda}(u) \psi,
$$

for every $\psi \in C_{0}^{\infty}(\Omega)$, with $\psi \geq 0$. Using again the chain rule,

$$
\nabla w=\nabla\left[H_{\lambda}(u)\right]=H_{\lambda}^{\prime}(u) \nabla u=-\frac{1}{\bar{f}_{\lambda}(u)} \nabla u
$$


and we obtain

$$
\int_{\Omega}-|\nabla u|^{2} \frac{\bar{f}_{\lambda}^{\prime}(u)}{\overline{f_{\lambda}(u)}} \psi-\int_{\Omega} \frac{\bar{f}_{\lambda}(u)}{\overline{f_{\lambda}(u)}} \nabla u \nabla \psi+\lambda \int_{\Omega} \frac{1}{w} \bar{f}_{\lambda}(u) \psi \frac{|\nabla u|^{2}}{\overline{f_{\lambda}(u)^{2}}} \geq \int_{\Omega} a \bar{f}_{\lambda}(u) \psi,
$$

for every $\psi \in C_{0}^{\infty}(\Omega)$, with $\psi \geq 0$. Since

$$
\lambda \frac{1}{w}=\bar{f}_{\lambda}^{\prime}\left(H_{\lambda}^{-1}(w)\right)=\bar{f}_{\lambda}^{\prime}(u)
$$

we deduce that

$$
-\int_{\Omega} \nabla u \nabla \psi \geq \int_{\Omega} a \bar{f}_{\lambda}(u) \psi, \quad \forall \psi \in C_{0}^{\infty}(\Omega), \quad \psi \geq 0,
$$

i.e. $u=H_{\lambda}^{-1}(w)$ is a subsolution of

$$
\Delta u=a \bar{f}_{\lambda}(u), \quad \text { in } \Omega .
$$

Corollary 2.6. Let $a \in L^{\infty}(\Omega)$ and assume that $g:(0,+\infty) \longrightarrow[0,+\infty)$ is a continuous function satisfying (1.1) and (1.2). If $w_{n}$ is a solution of $\left(P_{n}\right)$, then for every $\Omega_{0} \subset \subset \Omega$ there exists a constant $c_{\Omega_{0}}>0$ such that

$$
w_{n}(x) \geq c_{\Omega_{0}}, \quad \forall x \in \Omega_{0} .
$$

Proof. Consider $C$ given by (2.1). From (2.2) we have $h_{n}(s) \leq h(s) \leq 1 / s$, for every $s \in(0, C]$. This and the strict positiveness of $w_{n}$ (by Proposition 2.2) imply that $w_{n} \in H_{0}^{1}(\Omega)$ satisfies, in the weak sense,

$$
a(x)=-\triangle w_{n}+\lambda h_{n}\left(w_{n}\right)\left|\nabla w_{n}\right|^{2} \leq-\triangle w_{n}+\lambda \frac{1}{w_{n}}\left|\nabla w_{n}\right|^{2}, \quad x \in \Omega
$$

i.e. $w_{n}$ is a supersolution of

$$
\begin{gathered}
-\triangle w+\lambda \frac{1}{w}|\nabla w|^{2}=a(x), \quad x \in \Omega \\
w \in H_{0}^{1}(\Omega) .
\end{gathered}
$$

As $w_{n} \in H_{0}^{1}(\Omega) \cap C(\Omega)$, applying the Lemma $2.5, u_{n}=H_{\lambda}^{-1}\left(w_{n}\right)$ is a subsolution of

$$
\Delta u=a(x) \bar{f}_{\lambda}(u(x)), \quad \text { in } \Omega .
$$

Fix now $\Omega_{0} \subset \subset \Omega$. By Proposition 2.3, there exists $C_{\Omega_{0}}>0$ such that

$$
u_{n} \leq C_{\Omega_{0}}, \quad \forall x \in \Omega_{0}
$$

and thus, since $H_{\lambda}$ is nonincreasing,

$$
w_{n}=H_{\lambda}\left(u_{n}\right) \geq H_{\lambda}\left(C_{\Omega_{0}}\right) \equiv c_{\Omega_{0}}>0, \quad \forall x \in \Omega_{0} .
$$




\section{Positive solution for $(\mathrm{P})$}

In this section we study the convergence of the approximated solutions $w_{n}$ to a solution of $(P)$. We use the notation $w^{+}=\max \{w, 0\}, w^{-}=\min \{w, 0\}$ and for $k>0$, we also denote by $T_{k}$ and $G_{k}$ the real functions given by

$$
T_{k}(s):=\left\{\begin{aligned}
k, & s \geq k, \\
s, & -k \leq s \leq k, \quad G_{k}(s):=s-T_{k}(s), \quad s \in \mathbb{R} . \\
-k, & s \leq-k,
\end{aligned}\right.
$$

From Lemma 2.1, up to a subsequence, we can assume that

$$
w_{n} \rightarrow w, \text { in } H_{0}^{1}(\Omega) \text {. }
$$

By the $L^{\infty}$-estimate given in Lemma 2.1 and the almost everywhere convergence of $w_{n}$ to $w$ in $\Omega$ we get $w \in L^{\infty}(\Omega)$.

We are going to prove that for every $\omega \subset \subset \Omega,\left\{w_{n}\right\}$ converges to $w$ in $H_{0}^{1}(\omega)$. Specifically, we prove

\section{Proposition 3.1.}

$$
\lim _{n \rightarrow+\infty} \int_{\Omega}\left|\nabla\left(w_{n}-w\right)\right|^{2} \psi=0, \quad \forall \psi \in C_{0}^{\infty}(\Omega), \quad \psi \geq 0 .
$$

Remark 3.2. A consequence of the above proposition is that for every $\omega \subset \subset$ $\Omega$, there exists $h_{\omega} \in L^{2}(\omega)$ such that, up to a subsequence,

$$
\begin{aligned}
& \left|\nabla w_{n}\right| \leq h_{\omega}, \quad \text { a.e. } \omega, \\
& \nabla w_{n} \rightarrow \nabla w, \quad \text { a.e. } \omega .
\end{aligned}
$$

In particular, we have

$$
\nabla w_{n} \rightarrow \nabla w \quad \text { a.e. } \quad \Omega \text {. }
$$

Proof. Decompose $w_{n}-w$ in the way

$$
w_{n}-w=\left(w_{n}-T_{k}(w)\right)^{+}+\left(w_{n}-T_{k}(w)\right)^{-}-G_{k}(w) .
$$

We claim that for every $k>\|w\|_{\infty}$ and $\psi \in C_{0}^{\infty}(\Omega)$ with $\psi \geq 0$, it holds:

1. $\lim _{n \rightarrow+\infty} \int_{\Omega}\left|\nabla\left(w_{n}-T_{k}(w)\right)^{+}\right|^{2} \psi(x)=0$,

2. $\lim _{n \rightarrow+\infty} \int_{\Omega}\left|\nabla\left(w_{n}-T_{k}(w)\right)^{-}\right|^{2} \psi(x)=0$. 
For convenience of the reader, we postpone the proofs of these items to the next two lemmas and we use them here to conclude the proof of the proposition. Indeed, by the inequality

$$
\left|\nabla\left(w_{n}-w\right)\right|^{2} \leq\left|\nabla\left(\left[w_{n}-T_{k}(w)\right]^{+}\right)\right|^{2}+\left|\nabla\left(\left[w_{w}-T_{k}(w)\right]^{-}\right)\right|^{2}+\left|\nabla G_{k}(w)\right|^{2}
$$

we deduce that

$$
\begin{aligned}
\int_{\Omega}\left|\nabla\left(w_{n}-w\right)\right|^{2} \psi \leq & \int_{\Omega}\left|\nabla\left(\left[w_{n}-T_{k}(w)\right]^{+}\right)\right|^{2} \psi+\int_{\Omega}\left|\nabla\left(\left[w_{n}-T_{k}(w)\right]^{-}\right)\right|^{2} \psi \\
& +\int_{\Omega}\left|\nabla G_{k}(w)\right|^{2} \psi .
\end{aligned}
$$

Thus, given $\varepsilon>0$, by the convergence of $G_{k}(w)$ to 0 in $H_{0}^{1}(\Omega)$ as $k \rightarrow+\infty$, we can choose $k_{0}>\|w\|_{\infty}$ such that

$$
\left.\left|\int_{\Omega}\right| \nabla G_{k_{0}}(w)\right|^{2} \psi \mid \leq \varepsilon
$$

In consequence,

$$
\begin{aligned}
\limsup _{n \rightarrow+\infty} \int_{\Omega}\left|\nabla\left(w_{n}-w\right)\right|^{2} \psi \leq & \varepsilon+\lim _{n \rightarrow+\infty} \int_{\Omega}\left|\nabla\left(\left[w_{n}-T_{k_{0}}(w)\right]^{+}\right)\right|^{2} \psi \\
& +\lim _{n \rightarrow+\infty} \int_{\Omega}\left|\nabla\left(\left[w_{n}-T_{k_{0}}(w)\right]^{-}\right)\right|^{2} \psi
\end{aligned}
$$

(items 1. and 2.) $\leq \varepsilon$

from which the proof of Proposition 3.1 is concluded.

Now we prove in several lemmas the items 1. and 2. of the above proof.

\section{Lemma 3.3.}

$$
\lim _{n \rightarrow+\infty} \int_{\Omega}\left|\nabla\left(w_{n}-T_{k}(w)\right)^{+}\right|^{2}=0, \quad \forall k>\|w\|_{\infty} .
$$

Remark 3.4. Taking into account that

$$
\lim _{n \rightarrow+\infty} \int_{\Omega}\left|\nabla\left(w_{n}-T_{k}(w)\right)^{+}\right|^{2} \psi(x) \leq\|\psi\|_{\infty} \lim _{n \rightarrow+\infty} \int_{\Omega}\left|\nabla\left(w_{n}-T_{k}(w)\right)^{+}\right|^{2},
$$

item 1. in the proof of Proposition 3.1 is deduced from the above lemma. 
Proof. Denote $w_{n k} \equiv\left[w_{n}-T_{k}(w)\right]$. Using $w_{n k}^{+}$as test function in the equation satisfied by $w_{n}$,

$$
\int_{\Omega} \nabla w_{n} \nabla w_{n k}^{+}+\lambda \int_{\Omega} h_{n}\left(w_{n}\right)\left|\nabla w_{n}\right|^{2} w_{n k}^{+}=\int_{\Omega} a(x) w_{n k}^{+} .
$$

Since $w_{n} \geq 0$, we have $h_{n}\left(w_{n}\right) \geq 0$ and

$$
\int_{\Omega} \nabla w_{n} \nabla w_{n k}^{+} \leq \int_{\Omega} a(x) w_{n k}^{+}
$$

Adding and subtracting the term $\int_{\Omega} \nabla T_{k}(w) \nabla w_{n k}^{+}$we obtain:

$$
\int_{\Omega}\left|\nabla w_{n k}^{+}\right|^{2} \leq-\int_{\Omega} \nabla T_{k}(w) \nabla w_{n k}^{+}+\int_{\Omega} a(x) w_{n k}^{+} .
$$

The boundedness of $\left\{w_{n}\right\}$ in $H_{0}^{1}(\Omega)$ implies the boundedness of the sequence $\left\{w_{n k}^{+}\right\}$in $H_{0}^{1}(\Omega)$, and, up to a subsequence, for fixed $k$,

$$
w_{n k}^{+} \rightarrow\left(w-T_{k}(w)\right)^{+} \quad \text { as } \quad n \rightarrow+\infty .
$$

Then, passing to the limit in (3.1) as $n$ tends to infinity we get

$$
\lim _{n \rightarrow+\infty} \int_{\Omega}\left|\nabla w_{n k}^{+}\right|^{2} \leq-\int_{\Omega} \nabla T_{k}(w) \nabla\left(w-T_{k}(w)\right)^{+}+\int_{\Omega} a(x)\left(w-T_{k}(w)\right)^{+} .
$$

Observing that $\left(w-T_{k}(w)\right)^{+}$is 0 for $k>\|w\|_{\infty}$, we conclude that the right hand of the above inequality is tending to zero and the lemma is proved.

With respect to item 2. in the proof of Proposition 3.1, we show it in the sequel.

Lemma 3.5. For every $k>\|w\|_{\infty}$,

$$
\lim _{n \rightarrow+\infty} \int_{\Omega}\left|\nabla\left(w_{n}-T_{k}(w)\right)^{-}\right|^{2} \psi(x)=0 .
$$

Proof. Consider $\psi \in C_{0}^{\infty}(\Omega), \psi \geq 0$ and $\varphi_{\gamma}(s)=s e^{\gamma s^{2}}$ where the positive constant $\gamma$ will be chosen later in a suitable way. Denote, as in the above proof, $w_{n k} \equiv\left[w_{n}-T_{k}(w)\right]$. Since

$$
-k \leq w_{n k}^{-} \leq 0,
$$


610

D. Arcoya and P. J. Martínez-Aparicio

we have that $w_{n k}^{-} \in H_{0}^{1}(\Omega) \cap L^{\infty}(\Omega)$ and

$$
0 \geq \varphi_{\gamma}\left(w_{n k}^{-}\right) \psi \in H_{0}^{1}(\Omega) .
$$

Following [6] we use $\varphi_{\gamma}\left(w_{n k}^{-}\right) \psi$ as test function to deduce

$$
\begin{aligned}
& \int_{\Omega_{0}} \nabla w_{n} \nabla\left(w_{n k}^{-}\right) \varphi_{\gamma}^{\prime}\left(w_{n k}^{-}\right) \psi(x)+\int_{\Omega_{0}} \nabla w_{n} \nabla \psi(x) \varphi_{\gamma}\left(w_{n k}^{-}\right) \\
& +\int_{\Omega_{0}} \lambda h_{n}\left(w_{n}\right)\left|\nabla w_{n}\right|^{2} \varphi_{\gamma}\left(w_{n k}^{-}\right) \psi(x)=\int_{\Omega_{0}} a(x) \varphi_{\gamma}\left(w_{n k}^{-}\right) \psi(x),
\end{aligned}
$$

where $\Omega_{0} \subset \subset \Omega$ is such that supp $\psi \subset \Omega_{0}$. Adding and subtracting the term

$$
-\int_{\Omega_{0}} \nabla w \nabla\left(w_{n k}^{-}\right) \varphi_{\gamma}^{\prime}\left(w_{n k}^{-}\right) \psi(x),
$$

we get

$$
\begin{aligned}
& \int_{\Omega_{0}} \nabla\left(w_{n}-w\right) \nabla w_{n k}^{-} \varphi_{\gamma}^{\prime}\left(w_{n k}^{-}\right) \psi+\int_{\Omega_{0}} \nabla w \nabla\left(w_{n k}^{-}\right) \varphi_{\gamma}^{\prime}\left(w_{n k}^{-}\right) \psi \\
& \quad+\int_{\Omega_{0}} \nabla w_{n} \nabla \psi \varphi_{\gamma}\left(w_{n k}^{-}\right)+\int_{\Omega_{0}} \lambda h_{n}\left(w_{n}\right)\left|\nabla w_{n}\right|^{2} \varphi_{\gamma}\left(w_{n k}^{-}\right) \psi=\int_{\Omega_{0}} a \varphi_{\gamma}\left(w_{n k}^{-}\right) \psi .
\end{aligned}
$$

From Corollary 2.6,

$$
h_{n}\left(w_{n}\right)\left|\nabla w_{n}\right|^{2} \leq h\left(w_{n}\right)\left|\nabla w_{n}\right|^{2} \leq \frac{1}{c_{\Omega_{0}}}\left|\nabla w_{n}\right|^{2}, \quad \forall x \in \Omega_{0}
$$

and since $0 \geq \varphi_{\gamma}\left(w_{n k}^{-}\right) \psi$, we obtain by using $\nabla\left(w_{n}-w\right) \nabla w_{n k}^{-}=\left|\nabla w_{n k}^{-}\right|^{2}$ that

$$
\begin{aligned}
& \int_{\Omega_{0}}\left|\nabla\left(w_{n k}^{-}\right)\right|^{2} \varphi_{\gamma}^{\prime}\left(w_{n k}^{-}\right) \psi+\int_{\Omega_{0}} \nabla w \nabla\left(w_{n k}^{-}\right) \varphi_{\gamma}^{\prime}\left(w_{n k}^{-}\right) \psi \\
& \quad+\int_{\Omega_{0}} \nabla w_{n} \nabla \psi \varphi_{\gamma}\left(w_{n k}^{-}\right)+\int_{\Omega_{0}} \frac{\lambda}{c_{\Omega_{0}}}\left|\nabla w_{n}\right|^{2} \varphi_{\gamma}\left(w_{n k}^{-}\right) \psi \leq \int_{\Omega_{0}} a \varphi_{\gamma}\left(w_{n k}^{-}\right) \psi,
\end{aligned}
$$

i.e.

$$
\begin{aligned}
& \int_{\Omega_{0}}\left|\nabla\left(w_{n k}^{-}\right)\right|^{2} \varphi_{\gamma}^{\prime}\left(w_{n k}^{-}\right) \psi+\int_{\Omega_{0}} \frac{\lambda}{c_{\Omega_{0}}}\left|\nabla w_{n}\right|^{2} \varphi_{\gamma}\left(w_{n k}^{-}\right) \psi \\
& \quad \leq-\int_{\Omega_{0}} \nabla w \nabla w_{n k}^{-} \varphi_{\gamma}^{\prime}\left(w_{n k}^{-}\right) \psi-\int_{\Omega_{0}} \nabla w_{n} \nabla \psi \varphi_{\gamma}\left(w_{n k}^{-}\right)+\int_{\Omega_{0}} a \varphi_{\gamma}\left(w_{n k}^{-}\right) \psi .
\end{aligned}
$$


Observing that

$$
\begin{gathered}
\int_{\Omega_{0}}\left|\nabla w_{n k}^{-}\right|^{2} \varphi_{\gamma}\left(w_{n k}^{-}\right) \psi=\int_{w_{n}-T_{k}(w)<0}\left|\nabla w_{n k}\right|^{2} \varphi_{\gamma}\left(w_{n k}^{-}\right) \psi \\
=\int_{w_{n}-T_{k}(w)<0}\left|\nabla w_{n}\right|^{2} \varphi_{\gamma}\left(w_{n k}^{-}\right) \psi+\int_{w_{n}-T_{k}(w)<0}\left|\nabla T_{k}(w)\right|^{2} \varphi_{\gamma}\left(w_{n k}^{-}\right) \psi \\
-2 \int_{w_{n}-T_{k}(w)<0} \nabla w_{n} \nabla T_{k}(w) \varphi_{\gamma}\left(w_{n k}^{-}\right) \psi
\end{gathered}
$$

we derive

$$
\begin{aligned}
& \int_{\Omega_{0}}\left|\nabla\left(w_{n k}^{-}\right)\right|^{2}\left\{\varphi_{\gamma}^{\prime}\left(w_{n k}^{-}\right)+\frac{\lambda}{c_{\Omega_{0}}} \varphi_{\gamma}\left(w_{n k}^{-}\right)\right\} \psi \\
& \leq \frac{\lambda}{c_{\Omega_{0}}} \int_{w_{n}-T_{k}(w)<0}\left[\left|\nabla T_{k}(w)\right|^{2} \varphi_{\gamma}\left(w_{n k}^{-}\right) \psi-2 \nabla w_{n} \nabla T_{k}(w) \varphi_{\gamma}\left(w_{n k}^{-}\right) \psi\right] \\
& \quad-\int_{\Omega_{0}} \nabla w \nabla w_{n k}^{-} \varphi_{\gamma}^{\prime}\left(w_{n k}^{-}\right) \psi-\int_{\Omega_{0}} \nabla w_{n} \nabla \psi \varphi_{\gamma}\left(w_{n k}^{-}\right)+\int_{\Omega_{0}} a \varphi_{\gamma}\left(w_{n k}^{-}\right) \psi .
\end{aligned}
$$

For $k>\|w\|_{\infty}$ we have

$$
T_{k}(w)=w
$$

and by the Lebesgue dominated convergence theorem and the almost everywhere convergence of $w_{n}$ to $w$ in $\Omega$ we get

$$
\lim _{n \rightarrow \infty} \int_{w_{n}-T_{k}(w)<0}\left|\nabla T_{k}(w)\right|^{2} \varphi_{\gamma}\left(w_{n k}^{-}\right) \psi=\lim _{n \rightarrow \infty} \int_{\Omega_{0}} k \varphi_{\gamma}\left(w_{n k}^{-}\right) \psi=0 .
$$

In addition, if $\chi_{\Omega_{0}}$ denotes the characteristic function of the measurable set $\Omega_{0}$, this theorem also implies the following strong convergences in $L^{2}\left(\Omega_{0}\right)$ :

$$
\begin{gathered}
\nabla T_{k}(w) \varphi_{\gamma}\left(w_{n k}^{-}\right) \psi \chi_{\left\{w_{n}-T_{k}(w)<0\right\}} \rightarrow \nabla T_{k}(w) \varphi_{\gamma}\left(\left(w-T_{k}(w)\right)^{-}\right) \psi \chi_{\Omega_{0}} \stackrel{(3.4)}{=} 0 \\
\nabla w \varphi_{\gamma}^{\prime}\left(w_{n k}^{-}\right) \psi \chi_{\left\{w_{n}-T_{k}(w)<0\right\}} \longrightarrow \nabla w \varphi_{\gamma}^{\prime}\left(\left(w-T_{k}(w)\right)^{-}\right) \psi \chi_{\Omega_{0}}, \\
\nabla \psi \varphi_{\gamma}\left(w_{n k}^{-}\right) \chi_{\left\{w_{n}-T_{k}(w)<0\right\}} \longrightarrow \nabla \psi \varphi_{\gamma}\left(\left(w-T_{k}(w)\right)^{-}\right) \chi_{\Omega_{0}} \stackrel{(3.4)}{=} 0
\end{gathered}
$$


which together to the weak convergence of $\nabla w_{n}$ to $\nabla w$ (and thus of $\nabla w_{n k}$ to 0$)$ in $L^{2}\left(\Omega_{0}\right)$ gives us

$$
\lim _{n \rightarrow \infty} \int_{w_{n}-T_{k}(w)<0} \nabla w_{n} \nabla T_{k}(w) \varphi_{\gamma}\left(w_{n k}^{-}\right) \psi \chi_{\left\{w_{n}-T_{k}(w)<0\right\}}=0
$$

and

$$
\lim _{n \rightarrow \infty} \int_{\Omega_{0}} \nabla w \nabla w_{n k}^{-} \varphi_{\gamma}^{\prime}\left(w_{n k}^{-}\right) \psi=\lim _{n \rightarrow \infty} \int_{\Omega_{0}} \nabla w_{n} \nabla \psi \varphi_{\gamma}\left(w_{n k}^{-}\right)=0 .
$$

Therefore, by (3.5), (3.6) and (3.7), the inequality (3.3) shows that

$$
\lim _{n \rightarrow+\infty} \int_{\Omega_{0}}\left|\nabla\left(w_{n k}^{-}\right)\right|^{2}\left\{\varphi_{\gamma}^{\prime}\left(w_{n k}^{-}\right)+\frac{\lambda}{c_{\Omega_{0}}} \varphi_{\gamma}\left(w_{n k}^{-}\right)\right\} \psi=0 .
$$

Now, choosing $\gamma \geq\left(\frac{\lambda}{2 c_{\Omega_{0}}}\right)^{2}$, we have

$$
\varphi_{\gamma}^{\prime}(s)+\frac{\lambda}{c_{\Omega_{0}}} \varphi_{\gamma}(s)=e^{\gamma s^{2}}\left[1+2 \gamma s^{2}+s \frac{\lambda}{c_{\Omega_{0}}}\right] \geq \frac{1}{2}, \quad \forall s<0,
$$

and we deduce from the above limit that

$$
\lim _{n \rightarrow+\infty} \int_{\Omega_{0}}\left|\nabla\left(w_{n k}^{-}\right)\right|^{2} \psi=0
$$

for every $k>\|w\|_{\infty}$.

We conclude this section by showing that the limit $w$ of the approximated solutions $w_{n}$ is a solution for $(P)$, i.e. proving our Theorem 1.1:

Proof of Theorem 1.1. We can pass to the limit in the equation satisfied by the approximated solutions $w_{n}$ :

$$
\int_{\Omega} \nabla w_{n} \nabla \phi+\int_{\Omega} \lambda h_{n}\left(w_{n}\right)\left|\nabla w_{n}\right|^{2} \phi=\int_{\Omega} a \phi, \quad \forall \phi \in C_{0}^{\infty}(\Omega) .
$$

Indeed, for fixed $\phi \in C_{0}^{\infty}(\Omega)$, Proposition 3.1 (see also Remark 3.2) implies that

$$
\lim _{n \rightarrow+\infty} \int_{\Omega} \nabla w_{n} \nabla \phi=\int_{\Omega} \nabla w \nabla \phi
$$

On the other hand, from Corollary 2.6, there exists $c_{0}>0$ such that

$$
w_{n}(x) \geq c_{0}>0, \quad \forall x \in \omega \equiv \operatorname{supp} \phi .
$$


Thus

$$
h_{n}\left(w_{n}(x)\right) \leq h\left(w_{n}(x)\right) \leq \frac{1}{c_{0}}, \quad \forall x \in \omega
$$

and, again by Proposition 3.1, the dominated convergence theorem of Lebesgue implies

$$
\lim _{n \rightarrow+\infty} \int_{\Omega} \lambda h_{n}\left(w_{n}\right)\left|\nabla w_{n}\right|^{2} \phi=\int_{\Omega} \lambda h(w)|\nabla w|^{2} \phi .
$$

Therefore, passing to the limit as $n$ goes to infinity in the equation satisfied by $w_{n}$ we deduce

$$
\int_{\Omega} \nabla w \nabla \phi+\int_{\Omega} \lambda h(w)|\nabla w|^{2} \phi=\int_{\Omega} a \phi, \quad \forall \phi \in C_{0}^{\infty}(\Omega),
$$

i.e. $w \in H_{0}^{1}(\Omega)$ is a solution of $-\Delta w+g(w)|\nabla w|^{2}=a$ in $\Omega$.

On the other hand, as it has been observed, given $\omega \subset \subset \Omega$, by Corollary 2.6, $w_{n}(x)$ and thus $w(x)$ is away from zero for $x \in \omega$. Hence, [17, Theorem 1.1, p. 251] shows that $w \in C^{\alpha}(\bar{\omega})$, for every $\omega \subset \subset \Omega$, i.e. $w \in C^{\alpha}(\Omega)$.

As it was mentioned in the introduction we devote the last part of this paper to show how the previous arguments can be adapted to prove the Theorem 1.2.

Outline of the proof of the Theorem 1.2. For $q>\frac{N}{2}$, we consider $a \in L^{q}(\Omega)$ and the constant $C_{q}$ given by (2.3). Since, in this case, $g$ is continuous in $\left[0, C_{q}\right]$, there exists $M>0$ such that

$$
g(s) \leq M, \quad \forall s \in\left[0, C_{q}\right]
$$

We approximate the function $g$ by continuous functions $g_{n}: \mathbb{R} \rightarrow \mathbb{R}, n \in \mathbb{N}$ defined by

$$
g_{n}(s):= \begin{cases}g(s), & s \geq \frac{1}{n} \\ n^{2} s^{2} g(s), & 0<s \leq \frac{1}{n} \\ 0, & s=0, \\ -g_{n}(-s), & s<0 .\end{cases}
$$

Observe again that $g_{n}$ verifies

$$
\begin{array}{ll} 
& g_{n}(s) \stackrel{n \rightarrow+\infty}{\longrightarrow} g(s), \quad \forall s>0 \\
& g_{n}(s) \leq g(s), \quad \forall s>0, \\
\text { and } \quad & g_{n}(s) s \geq 0, \quad \forall s \in \mathbb{R} .
\end{array}
$$


Applying [6, Theorem 3.2, p. 43], there exists a nonnegative and nonzero solution $w_{n}$ of the approximated problem

$$
\begin{gathered}
-\Delta w+g_{n}(w)|\nabla w|^{2}=a(x) \text { in } \Omega \\
w \in H_{0}^{1}(\Omega) .
\end{gathered}
$$

From Lemma 2.1-i), $w_{n}$ is bounded in $H_{0}^{1}(\Omega)$ and in $L^{\infty}(\Omega)$. Specifically, it has been proved in this lemma that

$$
\left\|w_{n}\right\|_{L^{\infty}} \leq C_{q}, \quad \forall n \in \mathbb{N}
$$

Hence, we can assume that $w_{n}$ is weakly convergent to $w \in H_{0}^{1}(\Omega) \cap$ $L^{\infty}(\Omega)$. To prove the convergence of $w_{n}$ to $w$ in $H_{0}^{1}(\omega)$ for every $\omega \subset \subset \Omega$, we follow the same arguments to those in Proposition 3.1 by showing that

$$
\lim _{n \rightarrow+\infty} \int_{\Omega}\left|\nabla\left(w_{n}-T_{k}(w)\right)^{+}\right|^{2}=0,
$$

and

$$
\lim _{n \rightarrow+\infty} \int_{\Omega}\left|\nabla\left(w_{n}-T_{k}(w)\right)^{-}\right|^{2} \psi(x)=0 .
$$

The proof of (3.9) is deduced as in Lemma 3.3. On the other hand, the arguments of Lemma 3.5 can be adapted to obtain (3.10) without using the Corollary 2.6. Indeed, we just have to replace the estimate given in (3.2) by the following

$$
g_{n}\left(w_{n}\right)\left|\nabla w_{n}\right|^{2} \leq g\left(w_{n}\right)\left|\nabla w_{n}\right|^{2} \leq M\left|\nabla w_{n}\right|^{2}, \quad \forall s \in\left[0, C_{q}\right] .
$$

Consequently, we can assume that $w_{n} \rightarrow w$ in $H_{0}^{1}(\omega)$ for every $\omega \subset \subset \Omega$. To conclude it suffices to pass to the limit in the equation satisfied by $w_{n}$. This is done by applying similar arguments to those in the proof of Theorem 1.1. The only change is to replace (3.8) by $g_{n}\left(w_{n}(x)\right) \leq M$ for every $x \in \omega$.

\section{References}

[1] Abdellaoui, B., Dall'Aglio, A. and Peral, I.: Some remarks on elliptic problems with critical growth in the gradient. J. Differential Equations 222 (2006), 21-62.

[2] AlaA, N. and Mounir, I.: Weak solutions for some reaction-diffusion systems with balance law and critical growth with respect to the gradient. Ann. Math. Blaise Pascal 8 (2001), 1-19. 
[3] Bensoussan, A. and Boccardo, L.: Nonlinear systems of elliptic equations with natural growth conditions and sign conditions. Appl. Math. Optim. 46 (2002), 143-166.

[4] Bensoussan, A., Bocchrdo, L. and Murat, F.: On a nonlinear partial differential equation having natural growth terms and unbounded solution. Ann. Inst. H. Poincaré Anal. Non Linéaire 5 (1988), no. 4, 347-364.

[5] Boccardo, L., Murat, F. and Puel, J.-P.: Existence de solutions non bornées pour certaines équations quasi-linéaires. Portugal. Math. 41 (1982), $507-534$

[6] Boccardo, L., Murat, F. and Puel, J.-P.: Existence de solutions faibles pour des équations elliptiques quasi-linéaires à croissance quadratique. In Nonlinear partial differential equations and their applications. Collège de France Seminar, Vol. IV (Paris, 1981/1982), 19-73. Res. Notes in Math. 84. Pitman, Boston, Mass.-London, 1983.

[7] Boccardo, L., Murat, F. and Puel, J.-P.: Résultats d'existence pour certains problèmes elliptiques quasilinéaires. Ann. Scuola Norm. Sup. Pisa Cl. Sci. (4) 11 (1984), 213-235.

[8] Boccardo, L., Segura de León, S. and Trombetti, C.: Bounded and unbounded solutions for a class of quasi-linear elliptic problems with a quadratic gradient term. J. Math. Pures Appl. (9) 80 (2001), 919-940.

[9] Chincchio, F.: Regularity for solutions of nonlinear elliptic equations with natural growth in the gradient. Bull. Sci. math. 124 (2000), 57-74.

[10] Dall'Aglio, A., Giachetti, D. And Puel, J.-P.: Nonlinear elliptic equations with natural growth in general domains. Ann. Mat. Pura Appl. (4) 181 (2002), 407-426.

[11] Donato, P. And Giachetti, D.: Quasilinear elliptic equations with quadratic growth in unbounded domains. Nonlinear Anal. 10 (1986), 791804.

[12] Ferone, V. And Murat, F.: Nonlinear problems having natural growth in the gradient: an existence result when the source terms are small. Nonlinear Anal. 42 (2000), 1309-1326.

[13] Gilbarg, D. and Trudinger, N.S.: Elliptic partial differential equations of second order. Fundamental Principles of Mathematical Sciences 224. Springer-Verlag, Berlin, 1983.

[14] Grenon, N. and Trombetti, C.: Existence results for a class of nonlinear elliptic problems with $p$-growth in the gradient. Nonlinear Anal. 52 (2003), 931-942.

[15] Kazdan, J. L. And KrameR, R. J.: Invariant criteria for existence of solutions to second-order quasilinear elliptic equations. Comm. Pure Appl. Math. 31 (1978), 619-645.

[16] Keller, J. B.: On solutions of $\Delta u=f(u)$. Comm. Pure Appl. Math. 10 (1957), 503-510. 
[17] Ladyzhenskaya, O. A. And Ural'tseva, N. N.: Linear and quasilinear elliptic equations. Academic Press, New York-London, 1968.

[18] Landes, R.: On the existence of weak solutions of perturbated systems with critical growth. J. Reine Angew. Math. 393 (1989), 21-38.

[19] Miranda, C.: Su alcuni teoremi di inclusione. Ann. Polon. Math. 16 (1965), 305-315.

[20] Osserman, R.: On the inequality $\Delta u \geq f(u)$. Pacific J. Math. 7 (1957), 1641-1647.

[21] Porretta, A. And Segura de León, S.: Nonlinear elliptic equations having a gradient term with natural growth. J. Math. Pures Appl. (9) 85 (2006), 465-492.

[22] SERrin, J.: The problem of Dirichlet for quasilinear elliptic differential equations with many independent variables. Philos. Trans. Royal Soc. London Ser. A 264 (1969), 413-496.

[23] Stampacchia, G.: Équations elliptiques du second ordre à coefficients discontinus. Séminaire de Mathématiques Supérieures, 16 (Été, 1965). Les Presses de l'Université de Montréal, Montreal, Que., 1966

Recibido: 14 de noviembre de 2006

\author{
David Arcoya \\ Departamento de Análisis Matemático \\ Universidad de Granada \\ 18071-Granada, Spain \\ darcoya@ugr.es \\ Pedro J. Martínez-Aparicio \\ Departamento de Análisis Matemático \\ Universidad de Granada \\ 18071-Granada, Spain \\ pedrojma@ugr.es
}

Research supported by D.G.I. Project "Análisis no lineal y ecuaciones en derivadas parciales elípticas", MTM2006-09282 and Junta de Andalucía FQM-116. 